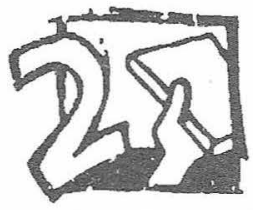

http://doi.org/10.15359/ree.2004-5.8

Educación Secundaria

\title{
DANIEL GALLEGOS Y EL CÍRCULO DE LA VIOLENCIA: PROPUESTA PARA EL ABORDAJE DEL GÉNERO DRAMÁTICO EN EL AULA
}

Nuria Méndez Garita*

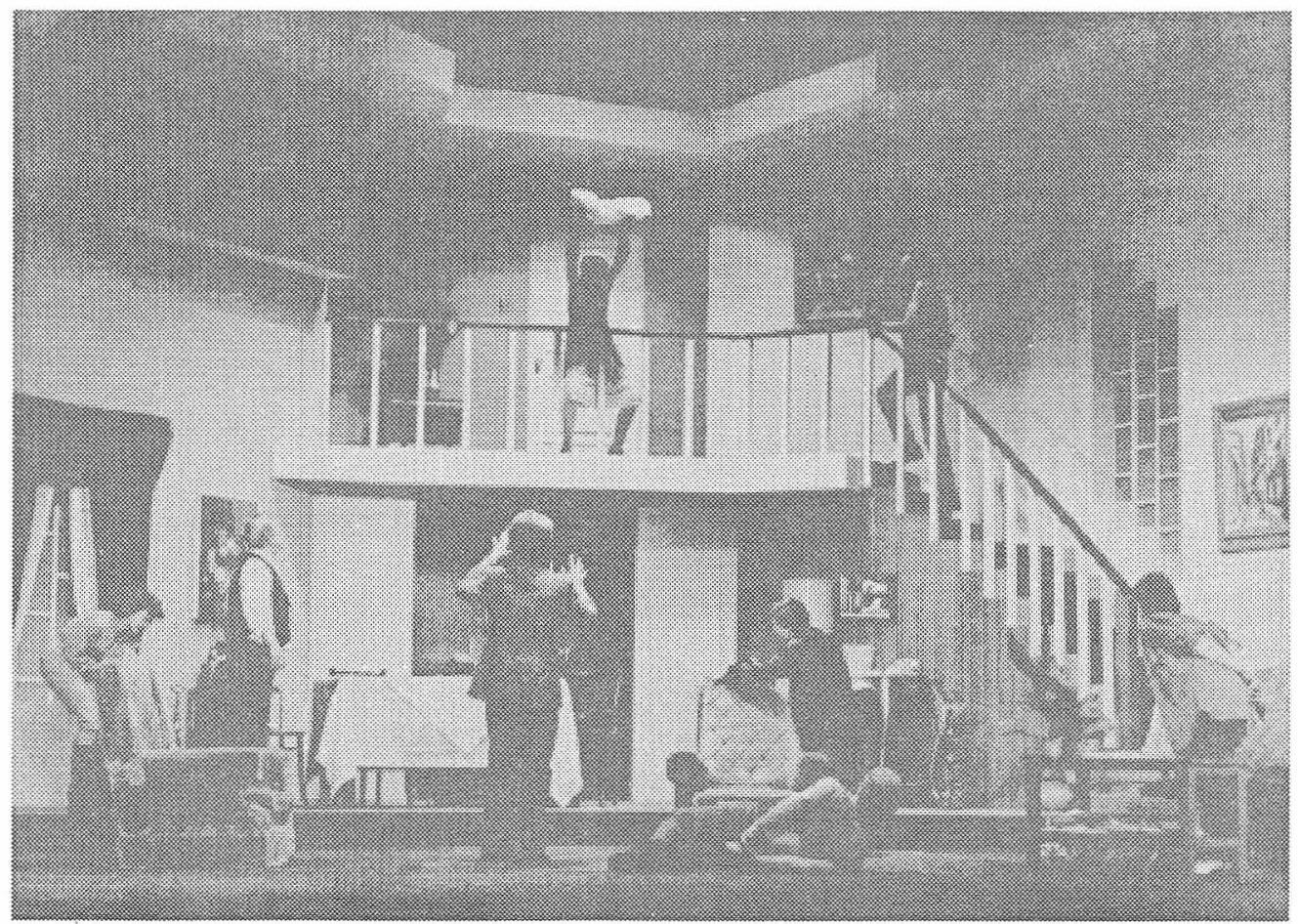

Dora: Tiraré al niño

Rưino: (Con el arma en la mano). Dame el niño... Si le hace algo al niño, le juro que los destruiré a todos. Nadie quedará vivo. Dora: (Triunfante) Nadie saldrá vivo de aquí tampoco.

(Actúan: Mariamalia Sotela, José Trejos, Miguel Calacci, Gladys Catania. Haydée de Lev. Mimi Prado. Leonardo Perucci y Lenín Vargas).

* Filóloga, graduada en la Universidad de Costa Rica. Actualmente, es candidata al grado de Maestría en Literatura Latinoamericana en ese mismo centro de estudios.

Académica de la División de Educación Rural. CIDE - UNA, desde el 2001. Tiene a su cargo los cursos de Literatura Infantil en esta unidad académica y la producción de material de apoyo para docentes rurales en el área de Español. Además, ha sido profesora de Español en varios colegios de Cartago y San José. 
La Literatura ofrece a los diferentes lectores posibilidades infinitas de significados. Permite reconstruir historias y mundos; proyectarse e imaginarse en diferentes repre. sentaciones, permite distanciarse para desGubrir la otredad v cada lector descubrirse u si mismo. La Literatura no existe para confortar o dar una postura equilibrada. Todo lo contrario. Su propósito es amenazar el equilibrio del lector: desestabilizarlo; conmoverlo. Hacerlo reflexivo.

El presente ensayo tiene varios fines: 1) acercarnos a un escritor costarricense de amplia trayectoria y cuy influencia en el teatro ha sido importante: Daniel Gallegos. 2) Proponer a los docentes una forma de análisis que facilite el estudio de la obra dramática y promueva la reflexión entre sus estudiantes.

Para tal efecto. se ha seleccionado la obra El séptimo círculo. ¿Por qué esta obra en especial? En estos tiempos, cuando el ser humano se ve inmerso en un ambiente convulso, cuando está a punto de perder la fe y la esperanza, es bueno detenerse y analizar su situación. Hay verdades complejas que se alojan en lugares terribles y la función inquietante del arte (en este caso específico, el teatro), es seguir recordándonos eso.
La violencia parece ser

el estado natural

de la humanidad.

Dr. Gutiérrez Gióngora

Estrenada en 1982 y publicada en 1991 por la Editorial Costa Rica, El séptimo círculo tiene por eje la violencia; ahí se sustentan los elementos dramáticos. Según Carmen Naranjo, esta obra "reveló el terrible signo de nuestros días. La escena es suceso de periódico y el suceso de periódico es escena, la calle es lección y el hecho cotidiano, cátedra." Para esta autora, el talento de Gallegos "puede ser el gran periodista que en el lenguaje teatral, nos golpee con la violencia en escena." (Gallegos, 1999).

La obra presenta escenas violentas que demuestran esadeshumanización del ser humano y que nos hace comprobar que el mundo es un lugar violento y peligroso. Cabe preguntarse entonces: ¿cómo se origina esa violencia?

Podemos contestarnos esta pregunta, si respondemos lo siguiente: $i$ acaso no es violencia que una madre o un padre utilicen como instrumento de chantaje a su propio hijo? ¿Acaso no es violencia que el esposo o compañero use a su mujer para conseguir sus fines? ¿Acaso la violencia no se genera porque vemos al otro como objeto y no como sujeto? ¿No se origina la violencia cuando menospreciamos las capacidades de los demás? ¿Acaso no hay más violencia cuando se refuerza la paz con la guerra? ¿Qué nos cuentan las noticias nacionales y mundiales?

El séptimo círculo, es una obra estructurada en dos actos, cuyo espacio físico es una casa de clase media alta. Gallegos nos ofrece una acción lineal y los acontecimientos progresan con gran rapidez hasta llegar al desenlace, culminación de hechos violentos. Los parlamentos son ágiles y los personajes que participan en ella, ven su personalidad modificada tras cada acción.

De un primer acto tranquilo y apacible, se pasa a un segundo cuya acción es más agresiva; los sucesos son más violentos, hay humillaciones y agresiones verbales y físicas. Son dos generaciones que se enfrentan: dos parejas de ancianos, 
Felix y Esperanza. Rodrigo y Dora por un lado, y cuatro delincuentes jóvenes, que no buscan robar, sino vengarse del los miembros que conforman su sociedad: Chita, Rufino, Manolo y Rona, esta aparece con un recién nacido en sus brazos.

El espacio psicológico está dominado por la incertidumbre y por la angustia, de principio a fin. Aquí es factible establecer una serie de oposiciones que circulan en la obra. Este hecho de una $u$ otra forma afecta al lector porque se pone en escena la violencia diaria en la que se vive. El peligro y la amargura que viene de afuera. La inseguridad social que lo invade y la duda ante cuáles pueden ser las actitudes del ser humano para con sus semejantes.

Esas oposiciones nos muestran enfrentadas a una generación mayor con otra más joven; la primera es una generación peligrosa para los jóvenes; los jóvenes son una amenaza para los mayores; la generación mayor tiene una condición de vida que le permite sentirse segura, cómoda; los jóvenes se sienten incómodos e inseguros y culpan de ello a sus antecesores. El futuro es sombrío e incierto.

Si dos generaciones se enfrentan, entonces, la sociedad es insegura. Si una generación desconfía de la otra, persiste la duda. Si vemos en el vecino el peligro, entonces, existe una amenaza. Si hay amenaza, puede haber violencia.

Y la violencia, que procede de la ira, es un deseo que nos incita, por odio, a hacer mal a quien odiamos. Y el odio es una tristeza acompañada por la idea de una causa exterior. Y la tristeza, no es otra cosa que el paso del ser humano de una mayor a una menor perfección.

\section{La intertextualidad en Gallegos}

La intertextualidad es lo que el nuevo texto reescribe del anterior. Implica una reelaboración y puede decirse que los textos se comunican entre sí, porque una palabra evoca a otra palabra, un personaje a otro.
Literature offers its readers infinite
possibilities in meaning. It allow's them to reconstruct stories and worlds. and to project or imaging themselves in various representations. I also allow's them to step away so as to discover the othemess and so that each reader can discover himself.

Literature does not exist to comfort us or to keep a balance. It is othenwise; its purpose is to threaten the reader's balance, to shake him. to move him deeply, and to make him reflect.

This essay has several objectives: I. to bring us near a Costa Rican writer of broad experience and who has had great influence in the country's theater: Daniel Gallegos: 2. to propose a form of analysis that will help students approach plays more easily and that will promote the reflection among them.

The analysis will be presented with the play El séptimo circulo (The Seventh Circle). This particular piece of writing was chosen because when humankind is immersed in such confusing times as we are now: when we are about to lose faith and hope. we need to stop and thing about the situation. Very complex truths can be found in terrible places and it is art's disturbing function (in this case, the theater) to keep reminding us of that. 


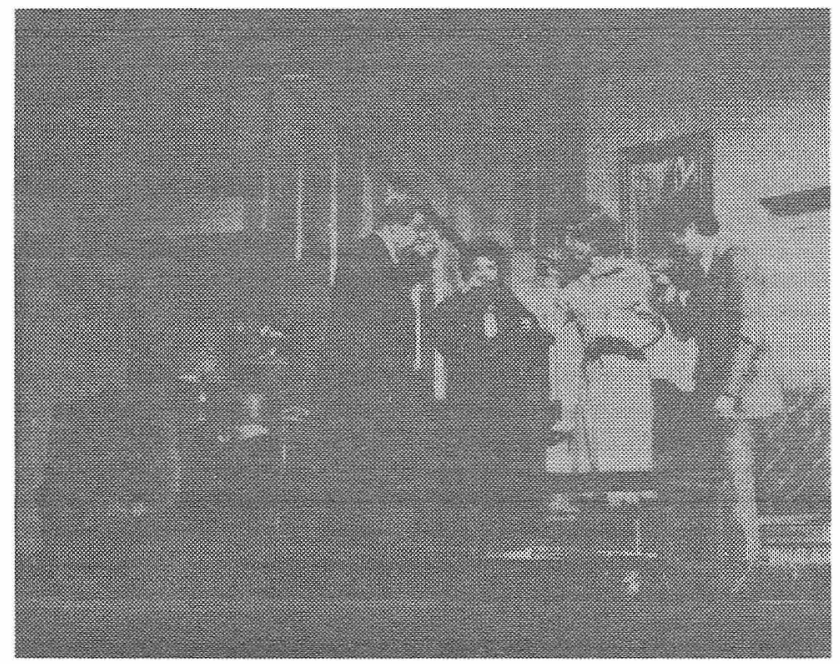

Félix: Bueno, a tomar este buen champagne y ¡a brindar!

Esperanza: ¡Por estar los cuatro juntos!

Rodrigo: ¡Por una vieja amistad!

(Actúan José Trejos. Gladys Catania, Haydée de Lev y Miguel Calacci).
Esta obra de Gallegos tiene como referente $\mathrm{La}$ Divina Comedia de Dante. Este poema mezcla la vida real con la sobrenatural; muestra la lucha entre la nada y la inmortalidad, una lucha donde se superponen tres reinos, tres mundos, logrando una suma de múltiples visuales que nunca se contradicen o se anulan. Los tres mundos Infiemo, Purgatorio y Paraíso reflejan tres modos de ser de la humanidad; en ellos se reflejan el vicio, el pasaje del vicio a la

virtud y la condición de los hombres perfectos. Es entonces a través de los viciosos, violentos y buenos que se revela la vida en todas sus formas, sus miserias y hazañas, pero también se muestra la vida que no es, la muerte, que tiene su propia vida, todo como una mezcla agraciada planteada por Dante, que se vuelve arquitecto de lo universal y de lo sublime.

Para efectos de este estudio, específicamente citamos El séptimo círculo, el de los violentos. Movidos por esa tristeza que achacan a causa externa, los iracundos, los violentos siembran la discordia mediante procedimientos fraudulentos y haciendo uso de una gran hipocresía.

El séptimo círculo en Dante, se divide en tres círculos llenos de piedra y rodeados por un gran río de sangre. A partir de este espacio, cada círculo empieza a tener divisiones que albergan una pena en particular, por ejemplo, los espíritus malditos que están divididos en tres: los violentos, los injuriosos y los usureros.

En el primer recinto del séptimo círculo, aparecen los violentos contra el prójimo. En el segundo recinto, los violentos contra sí mismos: los suicidas, los disipadores. Por último, en el tercer recinto del séptimo círculo: los violentos contra Dios, contra la naturaleza y contra la sociedad. Aquí están presentes los blasfemos, los sodomitas, los violentos contra la naturaleza y los usureros.

Estamos en los círculos de la maldad, que corresponde al acto humano cuyo objeto es afectar a otro ser humano, con daño físico en la persona o en sus cosas, o con el fraude que objetiva también un daño físico o moral. He aquí la 
reescritura del texto de Gallegos: hombre contra hombre; mujer contra mujer, hombre contra mujer; un niño objeto. Si leemos su obra con detenimiento, es notaria esa maldad, esa violencia que aumenta a cada instante: esa violencia que forma parte de la naturaleza humana.

\section{Conclusión}

En el séptimo círculo de Daniel Gallegos, es una obra que nos muestra, crudamente, la violencia de la que es capaz el ser humano, como también lo presentó Dante en su Infierno. Y muchas obras, además, contemplan este tema, que en Gallegos es recurrente. Lo encontramos también en su obra La casa, donde la connotación de casa/refugio se pierde ante la violencia que nace a lo interno de la misma, es decir, en la familia. (Rojas y Ovares,1993)

Encierro, violencia y opresión, siempre presentes en nuestra sociedad. Dos obras, dos épocas diferentes; dos escritores con un mismo tema. Tema eternamente humano: la violencia humana.

\section{Propuesta para un acercamiento a la obra teatral}

La finalidad del teatro es ofrecer una cuadro de la vida humana mediante la representación de una acción ficticia. Entre la sociedad y el teatro existen vínculos, pues este es reflejo de aquella.

El abordaje de los temas puede serle difícil al docente. No basta con solo leer teatro: hay que acercar al alumno al texto y lo más importante, mostrarle cuán importante es este género. Por eso y sin importar la obra seleccionada, la técnica del teatro forum promueve en el estudiante el sentido crítico ante diferentes hechos de la vida. Bien entendida esta técnica, lo acerca a la investigación, a la creatividad y a la discusión de temas polémicos $\mathrm{y}$ actuales.

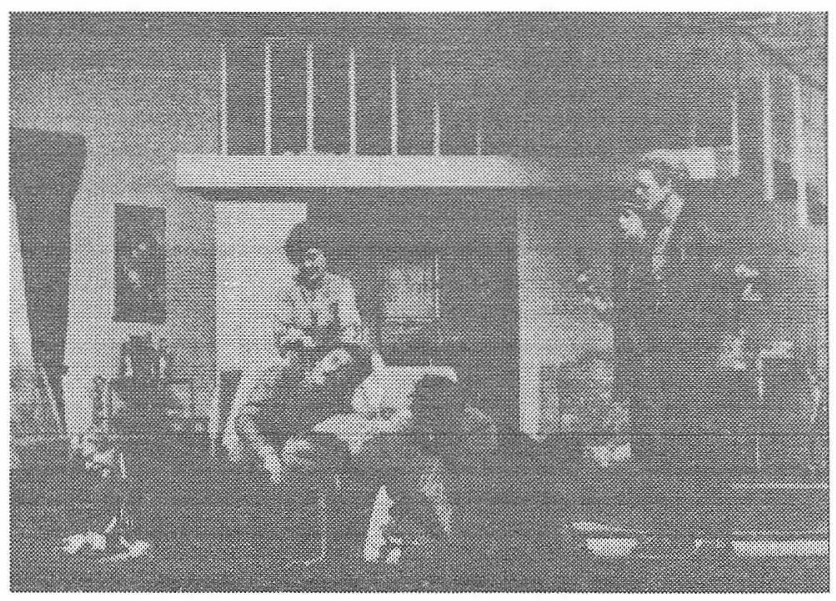

Rufino: ¡Con todo el carajo!: póngase en cuatro patas o le reviento las bolas a su marido.

(Rufino toma por la fuerza a Félix y lo obliga a ponerse de cuatro patas. El resto hace lo mismo. empujándolos, poniéndolos unos sobre otros).

Rufino: Ahora ladre... ¡Les digo que ladren!

(Actúan Mariamalia Sotela, Miguel Calacci, Haydée de Lev, Gladys Catania. Mimi Prado y Lenín Vargas). 


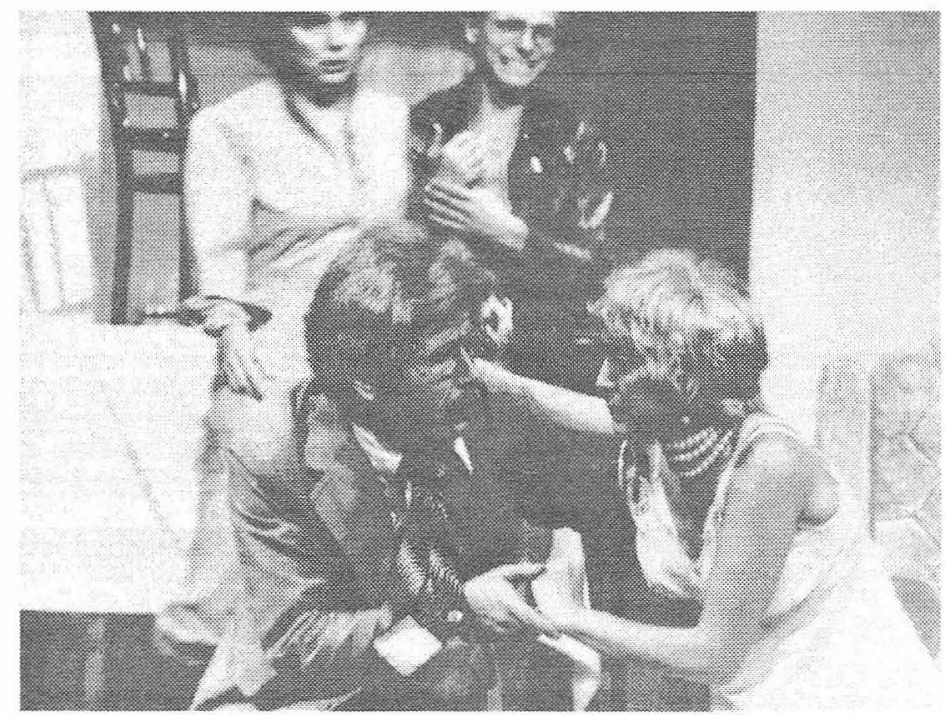

Dora: La verdad es que ya no me importa. Parece que ya se les olvidó cómo se huelen los perros y cómo se montan los unos a los otros... Pues a mí no se me ha olvidado. Rodrigo: Pero nosotros no vamos a actuar así.

Dora: ¿Qué nos impide?

(Actúan Miguel Callaci. Haydée de Lev. Mariamalia Sotela y Lenín Vargas).
Teatro-forum

¿Cómo hacer para que nuestros alumnos, además de leer la obra teatral, sean partícipes de ella como actores? ¿Cómo podemos acercarnos con ellos al análisis de este género literario, independientemente de cuál es la obra que corresponde leer según los programas de estudio? El teatroforum es una buena alternativa.

El teatro del forum, técnica emblemática del teatro del oprimido, es una herramienta importante con la que se envuelve al espectador haciéndolo participar de una acción dramática, en temas que lo motiven, como todo lo relacionado con el oprimido-opresor y juega con las misma reglas del cine-forum. A partir de la lectura de obra seleccionada, el docente establece los temas de análisis.

En esta técnica existe el comodín, que es el personaje que ejerce la función de mediador, clarifica las reglas entre el escenarioy el espectador; el comodín induce al debate y a una crítica política, social y global; también corrige, concilia y controla los términos del juego: la opresión y la violencia.

En una determinada situación escenificada en la que el espectador no se identifica con el desarrollo de los hechos, es decir, protesta, el comodín invita a los espectadores a entrar en escena sustituyendo al protagonista, y presentando alternativas para la solución de los conflictos planteados. El comodín auxilia la construcción del texto y el debate con las personas presentes, si las alternativas propuestas son la solución al problema, este será el final de la escena. El teatro-forum no tiene un final determinado y preescrito, ya que la intervención del público define el final.

\section{El método y los alumnos}

Apoyadas en el método expuesto, se puede trabajar en el aula para mejorar el proceso de enseñanza-aprendizaje tanto en el área de Español como en el 
área social. Como el teatro-forum facilita la reflexión y el análisis de diversos acontecimientos sociales presentes en el texto literario, puede ser un trabajo interdisciplinario y/o tener proyección comunal.

\section{¿Cómo lo hacemos?}

- Los alumnos pasan a formar parte del espectáculo.

- Se dividen en dos grupos: alumnos-espectadores y alumnosactores.

- El profesor cumple el papel del "comodín" como mediador y explica las reglas del juego.

- El profesor como mediador y educador controla el proceso, está siempre atento a la producción escrita y oral de la lengua, aplicada en la representación.

\section{Trabajo en clase}

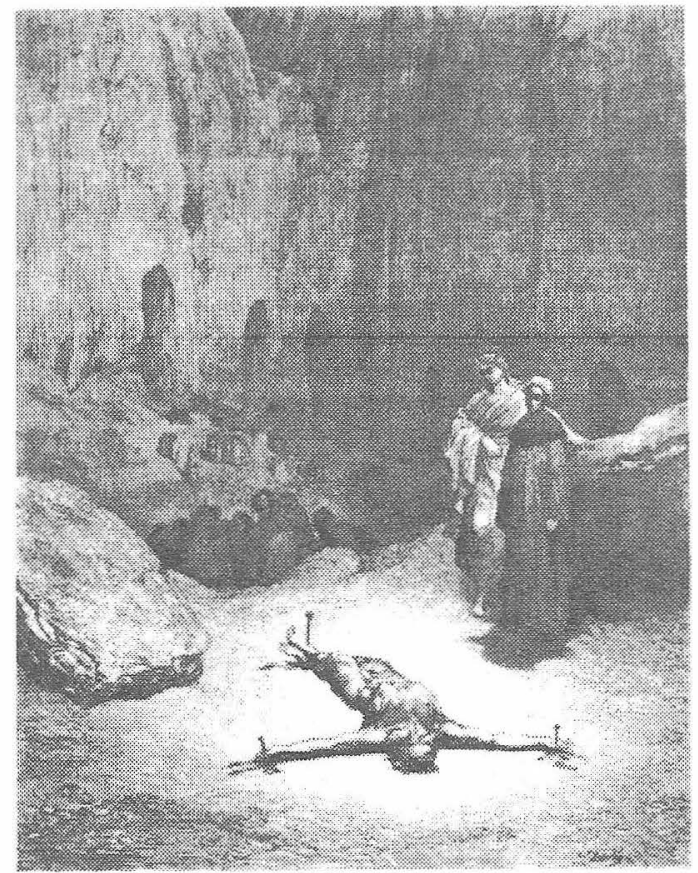

"Entonces, yo comencé a decirles: -Hermanos. los males que ustedes sufren...pero no pude continuar, porque vi delante a uno que estaba crucificado y clavado en el suelo con tres estacas. Al verme retorció todo su cuerpo..

-Ese que se encuentra en una cruz. fue el que aconsejó a los fariseos de que era conveniente llevar a un hombre al martirio..."

El Infierno, La Divina Comedia

Dante

El desarrollo de la actividad dentro de la clase, se realiza de la siguiente forma:

2.1 - En primer lugar incentivamos a los alumnos a una discusión sobre la temática expuesta en la obra y, a partir de ahí, la problemática social, discutiendo los hechos diarios, lo cotidiano; nosotros como profesores y educadores, orientamos la discusión. No obstante, respetando todas las opiniones, que inclusive siendo contrarias a nuestros objetivos, son de gran valor en el proceso y en la etapa final del trabajo: la puesta en escena (teatro-forum o sociodrama).

Podemos enriquecer la información y estimular la creatividad de los alumnos, si presentamos películas o elaboramos con ellos un collage con temas referentes a la discriminación (por homosexualismo, SIDA, género), donación de órganos, violencia y otros como noticias nacionales e internacionales (trabajo que puede ser coordinado con los departamentos de Orientación y Estudios Sociales y Cívica). Todo el material presentado debe ser revisado, con lo cual apoyamos el vocabulario, la interpretación, la comprensión auditiva y la crítica. 
2.2- En esta segunda etapa, comenzamos a formar dos equipos de trabajo e investigación, aquí ya los alumnos tienen un conocimiento del tema.

En esta etapa, los equipos escogen un tema y adoptan una posición ante este; en este punto se comienza a trabajar la comprensión escrita y oral, en la que los propios alumnos organizan los textos (guión para la representación, o bieri. ensayos breves con los temas que se representan). Se puede urur el tema con el proceso de investigación y la presentación del informe escrito.

2.3 - En esta tercera etapa, los equipos se juntan en un gran taller; el profesor coordina el trabajo, explica las reglas del juego, corrige los errores en la producción escrita, oral y de montaje escénico.

Este trabajo puede realizarse varias veces con temas diferentes e intercambiando los equipos y posiciones en escena, así como los alumnos dentro de los equipos; de esta forma trabajamos la interactividad, lo cognitivo y la disciplina (fundamental en el proceso).

En el decorrer de los talleres, siempre debemos dejar tiempo para trabajar la expresión corporal, la voz, la memorización, organizar juegos teatrales, sin olvidar que nuestra propuesta está basada en el teatro-educación, y que la finalidad de este método es la puesta en escena con todos sus componentes didácticos y pedagógicos, así como el hecho de generar en los estudiantes un espíritu crítico.

El docente administra el tiempo, las tareas en clase y guía la discusión en torno a la problemática tratada. Es importante enfatizar en el respeto por las ideas y posiciones de los alumnos.

\section{Objetivos}

Como todo trabajo, la planificación de esta actividad es vital, máxime si se logra como un trabajo interdisciplinario. Los objetivos se redactan según la temática propuesta y la importancia del análisis de este género.

\section{Evaluación}

La evaluación debe tener en cuenta el desarrollo y capacidad de cada uno de los alumnos y estar en relación con la finalidad de las funciones linguiísticas.

A la hora de evaluar la actividad, es conveniente que el docente construya su propia escala de valores que contemple, entre otros aspectos:

- El grado de incorporación de los conocimientos lingüísticos de la lengua española.

- Integración al equipo de estudio, para el logro de los objetivos propuestos.

- Valorización de la capacidad creadora y el crecimiento del alumno como individuo. 
- La puesta en escena, con todos sus elementos integradores, pedagógicos y didácticos.

Todo docente puede ayudar al desarrollo de la capacidad creadora de los alumnos. Para ello existen diferentes técnicas. El teatro-forum es una más que combina una serie de elementos y hace de las lecciones un proceso dinámico. pues es un instrumento didáctico que permite acercar a los alumnos a la reflexión en torno a cierta problemática que nos afecta como sociedad. El punto de arranque es la obra literaria.

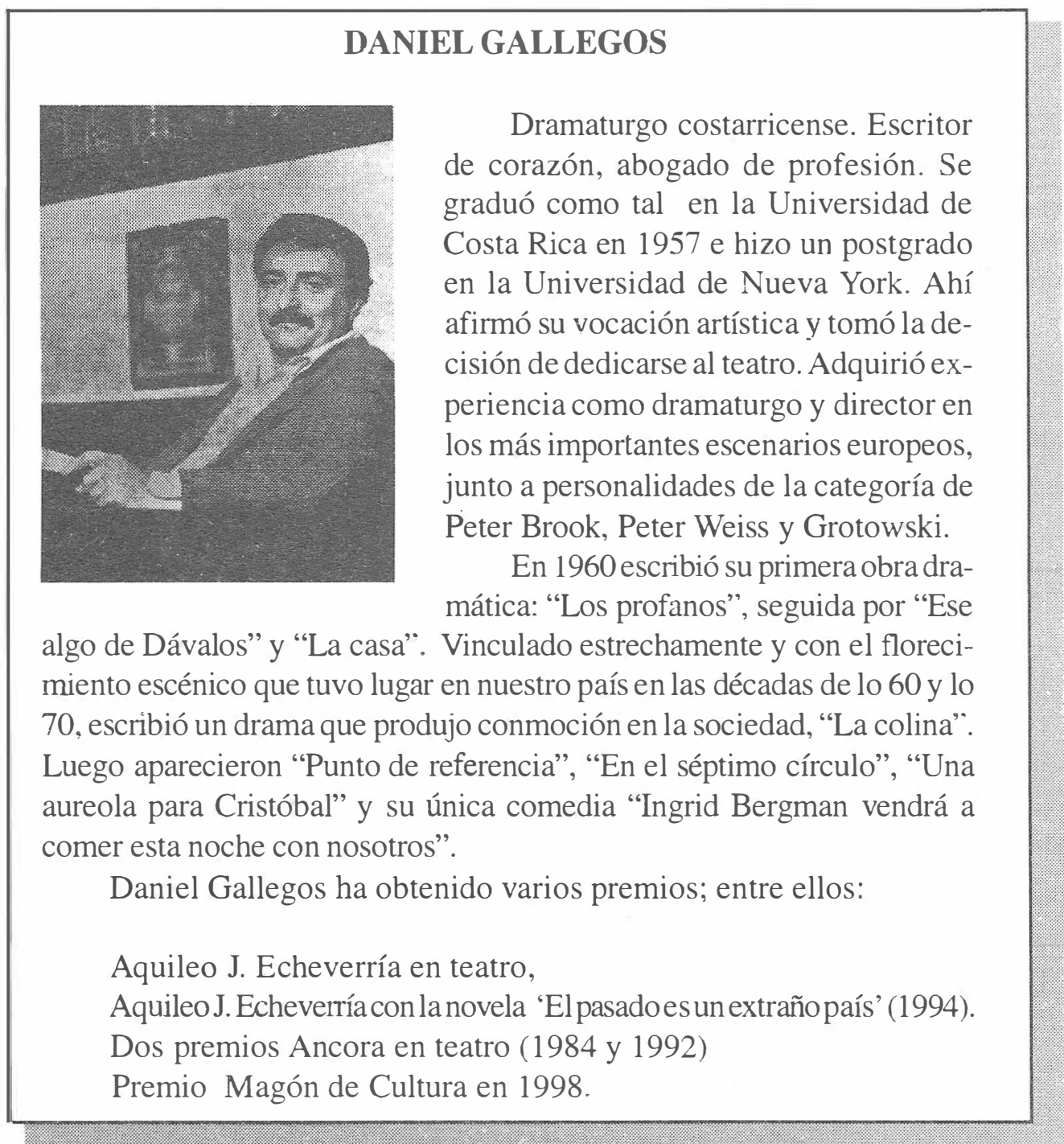




\section{Referencias}

Dante Alighieri, (1998) La Divina Comedia. Grandes genios de la literatura universal; España, 1998.

Dante Alighieri. (2002) La Divina Comedia. El Infierno. Ilustraciones de Gustavo Doré. Grupo Editorial Tomo, S.A., México.

Gallegos, Daniel. (1999) La casa y otras obras. Editorial Costa Rica, San José.

Pérez-Rioja, J.A. (1977) Diccionario de literatura universal. Editorial Tecnos, Madrid, España.

Rojas, Margarita y Flora Ovares. (1993) 100 años de la literatura costarricense. Editorial Norma. San José, Costa Rica.

http://www.lamaga.com.ar/www/area2/pg_nota.asp?id_nota=875

http://www.servisur.com/cultural/dante/comediainf/notdcil111.htm

http://www.henciclopedia.org.uy/autores/Rehermann/Silencio.htm

http://www.celis.info/trabajos/divinacomediainfierno.htm

www.udec.cl/ docliter/portada2_archivos/teayedu.htm

http://members.tripod.com/ thcincost/Teatro.htm

http://www.montfort.org.br/esp/veritas/numero.html

http://www.deon.com.ar/63kirov.htm

http://www.enciclopediacatolica.com/n/numeriglesia.htm 